\title{
Ampullary cancer in a patient with familial adenomatous polyposis - a rare case report and current status of management
}

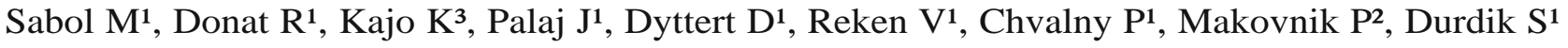 \\ Department of Surgical Oncology, Faculty of Medicine Comenius University and St. Elisabeth Cancer Center, \\ Bratislava, Slovakia. martin.sabol@ousa.sk
}

\begin{abstract}
Familial adenomatous polyposis (FAP) is an inherited autosomal dominant disorder. Extracolonic manifestations are seen quite often. As prophylactic colectomy has become a standard care in FAP patients, the concerns over the development of associated extracolonic malignancies have become more prevalent. The authors report a case of a patient with the history of subtotal colectomy because of FAP with the development of adenocarcinoma of papilla of Vater twenty-six years later. A radical procedure in form of proximal pancreaticoduodenectomy was indicated. Variable endoscopic surveillance protocols and treatment strategies have been proposed concerning the management of duodenal and periampullary lesions. In case of periampullary malignancies, the radical surgical resection offers the only chance for cure and the only option that may safeguard the long-term survival (Fig. 2, Ref. 30). Text in PDF www.elis.sk.

KEY WORDS: ampulla of Vater, bile duct, obstructive jaundice, pancreatoduodenectomy, periampullary tumors.
\end{abstract}

\section{Introduction}

Familial adenomatous polyposis (FAP) is an inherited autosomal dominant disorder characterized by numerous colonic polyps that represent obligatory precancerous lesions. Extracolonic manifestations are seen quite often. Patients with FAP often present with periampullary adenomas that can transform into periampullary cancer. (1) Persons suffering from FAP may have a 100 to 200fold increased risk of the occurrence of periampullary carcinoma. (2) Radical surgical resection offers the only chance for cure and the only option for safeguarding the long-term survival in patients with periampullary malignancies. A local excision of periampullary cancer should be reserved for patients unfit for radical resection. The endoscopic treatment of duodenal lesions is controversial but it seems to be a safe and efficient procedure for adenomas with low to moderate dysplasia and in selected patients with severe duodenal polyposis it can serve as an alternative to surgery.

\section{Case report}

We present a case of a fifty-eight-year old male patient with the history of subtotal colectomy with J-pouch formation conducted in 1988 because of FAP. In his case, obstructive jaundice with cholangitis occurred in November 2014. The endoscopic examination

${ }^{1}$ Department of Surgical Oncology, Faculty of Medicine Comenius University and St. Elisabeth Cancer Center, Bratislava, Slovakia, ${ }^{2}$ Department of Gastroenterology, St. Elisabeth Cancer Center, Bratislava, Slovakia, and ${ }^{3}$ Department of Pathology and Cytology. St. Elisabeth Cancer Center, Bratislava, Slovakia

Address for correspondence: M. Sabol, Department of Surgical Oncology, St. Elisabeth Cancer Center, Bratislava, Slovakia.

Phone: +421.2.39249281 revealed hyperplastic polypoid papilla of Vater with high grade of dysplasia. Endoscopic implantation of duodenobiliary stent was performed. As a result, the jaundice regressed. The duodenobiliary stent was then replaced twice and during the endoscopic control in June of 2015, a markedly progressed polypoid lesion of papilla of Vater was revealed. Repeated biopsy showed an infiltration of the papilla with the intestinal type of adenocarcinoma, G1-2. The preoperative CT staging and MRCP confirmed the presence of tumor of the papilla of Vater with a "double-duct sign" and extensive regional lymphadenopathy (Fig. 1). In October 2015, the patient underwent a radical surgical resection of proximal pancreaticoduodenectomy with extensive lymphadenectomy which was performed at the Department of Surgical Oncology, Saint

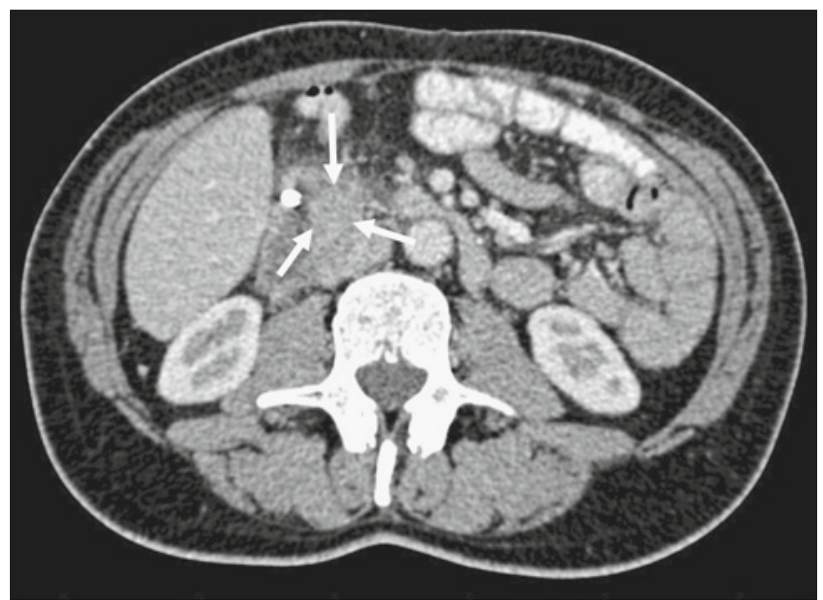

Fig. 1. CT scan showing tumor of the ampulla of Vater with extensive regional lymphadenopathy. 


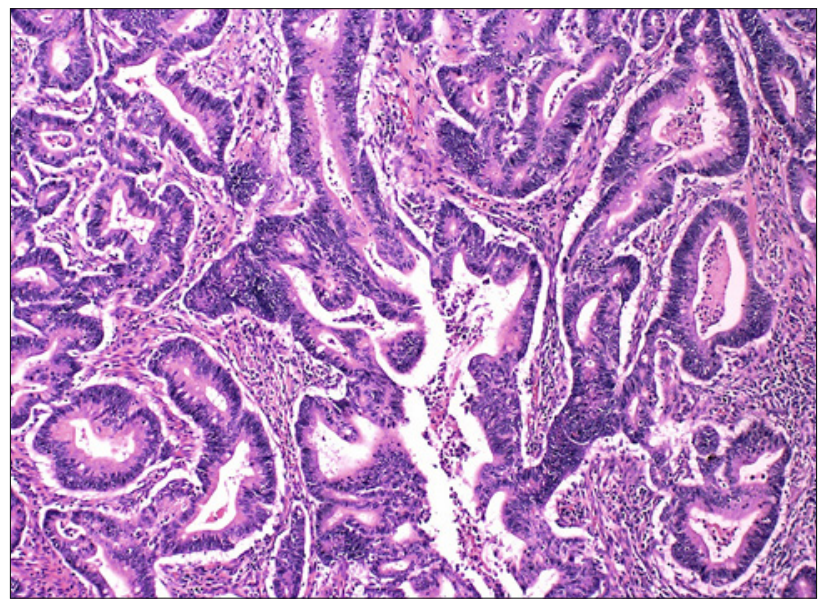

Fig. 2. Histopathologic specimen showing an intestinal type of well-differentiated invasive adenocarcinoma of the ampulla of Vater, (hematoxyllin and eosin staining, 200x magnification).

Elisabeth Cancer Center in Bratislava. The reconstruction was achieved by duct-to-mucosa pancreaticojejunostomy, hepaticojejunostomy, gastroenterostomy and jejunojejunostomy. The histopathologic examination confirmed a well-differentiated intestinal type of invasive adenocarcinoma of the ampulla of Vater (Fig. $2)$. No metastases in retrieved regional lymph nodes were found (pT2pN0(0/25)M0). The postoperative course was uneventful; the patient was discharged on the 12th postoperative day. Because of the absence of negative prognostic factors, an adjuvant systemic treatment was not indicated.

\section{Discussion}

Familial adenomatous polyposis is an autosomal dominantly inherited disorder caused by germline mutations in the APC gene with a nearly complete penetrance of the colonic phenotype but with a variable penetrance of extraintestinal manifestations of the disease (3). In the classic form of FAP, colonic polyposis develops in over $90 \%$ of affected individuals by the age 35 . The lifetime risk of colorectal cancer is nearly $100 \%$ in the absence of colectomy. The duodenum is the second most common site of polyp formation. Observed in up to $90 \%$ of individuals with FAP, duodenal polyps are usually recognized 10-20 years after the diagnosis of colonic polyposis. Duodenal/periampullary adenocarcinoma is the second most common cause of cancer death in FAP patients and the cumulative risk of duodenal cancer is estimated to be as high as $10 \%$ by age of 60 years, which is $100-200$-fold higher than in the general population (4).

The severity of duodenal polyposis is assessed by the Spigelman classification (5).

As prophylactic colectomy has become a standard care in FAP patients, the concerns over the development of associated extracolonic malignancies have become more prevalent (6).

Surgical options for FAP patients comprise total proctocolectomy with permanent ileostomy, colectomy with ileorectal anas- tomosis or extensively used restorative proctolectomy with the formation of ileoanal pouch (7). Adenocarcinoma of the ampulla of Vater is the second most common periampullary adenocarcinoma, representing $21 \%$ of patients undergoing Whipple's resection (8). Tumors of the ampulla of Vater are relatively rare, with an estimated incidence of 5.7 per million population per year, representing $0.2 \%$ of gastrointestinal tract malignancies and $7-12 \%$ of periampullary tumors $(9,10)$. Microscopic adenomatous changes are relatively common in FAP patients. Burke et al. noted that 54 $\%$ of FAP patients harbored ampullary adenomas even when the ampullae had a normal appearance on direct examination (11). Periampullary cancers constitute a distinct entity compared to that of the classic pancreatic head cancer and carry a better prognosis than adenocarcinoma of the pancreatic head (12). Concerning periampullary malignancies, the radical surgical resection offers the only chance for cure and the only option of safeguarding the long-term survival. Pancreaticoduodenectomy (PD; Whipple operation,) represents the procedure of choice for these malignant tumors. Local excision should only be attempted in patients with small adenocarcinoma of the ampulla of Vater, who are considered unfit or who refuse radical resection. In these subgroups of patients, a 5-year survival rate of $40 \%$ has been reported (13).

As the duodenal cancer has become an increasingly important cause of morbidity and mortality in patients with FAP, the management of duodenal adenomatosis as a precancerosis remains the major challenge to the gastroenterologist in these patients. Transduodenal excision used to be advocated as the procedure of choice but it has a high recurrence rate. Surgical ampullectomy is an alternative for patients with ampullary polyps for whom endoscopic treatment is not safe (14). Because radical surgical Whipple procedure could be associated with significant morbidity, the decision-making represents a difficult process during which the risk/benefit ratio of surgical intervention should be considered. Currently, no data concerning this issue are available. The prognosis of duodenal cancer in patients with FAP depends on its early surgical removal, which justifies the aggressive approach to advanced benign adenomatosis (15). Thus, an effective surveillance is recommended to identify risk lesions before the malignant transformation appears. For this reason, some centers offer surgical treatment right after the diagnosis of an advanced benign disease (stages III or IV) (16).

Nevertheless, even extensive procedures do not entirely eliminate the risk of cancer. The endoscopic follow-up has documented recurrent adenomas and even cancer in the proximal small bowel after duodenectomy (17).

Variable endoscopic surveillance protocols and treatment strategies have been proposed, including routine biopsies of the papilla even if it appears macroscopically normal (18). The endoscopic treatment of duodenal lesions remains controversial; their removal is associated with the risk of perforation or pancreatitis. Open polypectomy via duodenotomy is linked with high recurrence rate. The primary indication for surgery is assessed in cases of progressive duodenal polyposis (Spigelman stage IV) (19). The recurrence rate of duodenal lesions after endoscopic intervention remains high. However, endoscopic surveillance with directed endotherapy for 
908-911

duodenal lesions in FAP patients is feasible and safe when diligently performed (20). Ampullary tumors have a quite good prognosis as they are often resectable. The absence of perioperative mortality, and acceptable morbidity rate in HPB centers along with low incidence of benign tumors among resected patients support the aggressive surgical approach to the management of ampullary tumors (21). The factors of very low prevalence of periampullary cancer in patients with FAP, lack of published data and difficulty of pancreatic surveillance for pancreatic neoplasia contribute to the fact that no recommended pancreatic surveillance protocol has been made yet. Also, the identification rate of patients with the classic variant of exocrine pancreatic adenocarcinoma in patients with FAP is extremely rare; they are a rather unusual type of tumors. The relationship between FAP and pancreatic/periampullary tumors is controversial and so far, no formal risk assessment of these extraintestinal malignancies in FAP has been reported (22). Mutations in BRCA2 confer a higher risk for developing cancers of the pancreas. BRCA2 mutations were observed with a frequency of $14.3 \%$ in association with ampulla of Vater carcinomas (23). The relative risk of cancer transformation of ampullary versus nonampullary duodenal polyps in FAP patients is not clear but some studies report an increased risk of carcinoma in ampullary compared with nonampullary adenomas. The risk increases with the size of adenoma, villous histology or moderate/severe dysplasia (24). The malignant progression of duodenal adenomas is slow and proceeds with the accumulation of genetic mutations (such as APC, k-RAS, p53) in a stepwise manner analogous to colorectal polyps. In general, the prognosis of ampullary and duodenal cancers in these patient groups is poor (25).

Nevertheless, there is no clear genotype-phenotype relationship with duodenal cancer in FAP. This is consistent with most studies assessing the duodenal disease in FAP.

The assessment of risk of duodenal cancer would probably require to update the Spigelman classification system. Recent advances in endoscopic equipment and techniques enable to detect a greater number of small duodenal lesions resulting in Spigelman classification upstaging. But it does not generally corelate with the increased cancer risk (26).

It has to be emphasized that ampullary and duodenal lesions should be considered separately as well as staged differently. Neither Spigelman classification itself nor biopsies taken from suspicious advanced lesions may reveal the presence of carcinoma, and upstaging is confirmed only postoperatively (27). It seems to be evident that patients with the greatest risk of development of periampullary carcinoma are presumed to be those with Spigelman stage IV disease. The course of the development of periampullary cancer from stage IV disease ranged from 0.5 to 9 years. Burke reports that none of these adenocarcinomas were detected during endoscopic surveillance which was performed over a median of 16 months before the detection of cancer. It suggests that cancerous duodenal lesions could be missed during endoscopic surveillance and thus allowed to sneak through unnoticed and progress rapidly (28).

After reaching the fifth decade of life, FAP patients must be advised about the risks of developing a duodenal cancer. In fact, the surveillance should start after 25 years of age. When necessary, radical procedure such as PD may be performed with acceptable morbidity by skilled surgical teams (29).

Despite significant advances in genetic analyses of FAP in last decades, the mortality from periampullary cancer has remained virtually unchanged. The identification of genotypic factors that predict which patients are at greatest risk of development of periampullary carcinoma would allow for a more rational approach to the clinical management of duodenal disease in FAP (30).

\section{Conclusion}

FAP is one of the best-defined hereditary cancers that account for less than $1 \%$ of all CRC cases. Untreated polyposis leads to $100 \%$ risk of cancer. When considering the prophylactic colectomy as a standard approach in the management of the patients with FAP, duodenal cancer in these patients has become a major cause of mortality. Duodenal lesions represent a common finding in patients with familial adenomatous polyposis and screening for duodenal adenocarcinoma is currently recommended. Variable endoscopic surveillance protocols and treatment strategies have been proposed, including routine biopsies of the papilla even if it appears macroscopically normal. Ampullary and duodenal diseases should be considered separately and staged differently. Surgical resection represents the only curative treatment option for periampullary malignancies but radical surgical procedure could be associated with significant morbidity.

\section{References}

1. Stolte M, Pscherer C. Adenoma-carcinoma sequence in the papilla of Vater. Scand J Gastronterol 1996, 31: 376-382

2. Pauli RM, Pauli ME, Hall JG. Gardner syndrome and periampular malignancy. Am J Med Genet 1980, 6: 205-219.

3. Dobbie $\mathbf{Z}$ et al. Correlation between the development of extracolonic manifestations in FAP patients and mutations beysond codon 1403 in the APC gene. J Med Gent 1996; 33: 274-280.

4. Bjork J, Akerbrant H, Iselius L, Bergman A, Engwall Y, Wahlström J, Martinsson T, Nordling M, Hultcrantz R. Periampullary adenomas and adenocarcinomas in familial adenomatous polyposis. Gastroenterology 2001; 121 (5): 1127-1135.

5. Spigelman AD, Williams CB, Talbot IC, Domizio P, Phillips RK. Upper gastrointestinal cancer in patients with familial adenomatous polyposis. Lancet 1989; 2: 783-785.

6. Attard TM, Cuffari C, Tajouri T, Stoner JA, Eisenberg MT, Yardley JH et al. Multicenter experience with upper gastrointestinal polyps in pediatric patients with familial adenomatous polyposis. Am J Gastroenterol 2004; 99: 681-686.

7. Tudyka VN, Clark SK. Surgical tretament in familial adenomatous polyposis. Ann Gastroenterol 2012; 25 (3): 201-2016

8. Crist DW, Sitzmann JV, Cameron JL. Improved hospital morbidity, mortality, and survival after the Whipple procedure. Ann Surg 1987; 206: 358-365.

9. Neoptolemos JP, Talbot IC, Carr-Locke DL, Shaw DE, Cockleburgh R, Hall AW et al. Treatment and outcome in 52 consecutive cases of ampullary carcinoma. Br J Surg 1987; 74: 957-961. 
10. Howe JR Klimstra DS, Moccia RD, Colon KC, Brennan MF. Factors predictive of survival in ampullary carcinoma. Ann Surg 1997; 228: 97-94.

11. Burke CA, Beck GJ, Church JM, van Stolk RU. The natural history of untreated duodenal and ampullary adenomas in patients with familial adenomatous polyposis followed in an endoscopic surveillance program. Gastrointest Endosc 1999; 49: 358-364.

12. Jagannath $P$, Shrikhande $S$. Current options in the diagnosis and management of periampullary carcinoma. Indian J Surg 2003; 65 (4): 347-353.

13. Fernandez-Cruz L. Periampullary carcinoma in Surgical Treatment. Evidence-Based and Problem-Oriented.Holzheimer RG, Mannick JA. Munich: Zuckschwerdt, 2001.

14. Cordero-Fernández C, Garzón-Benavides M, Pizarro-Moreno A, García-Lozano R, Márquez-Galán JL, López Ruiz T. Gastroduodenal involvement in patients with familial adenomatous polyposis. Prospective study of the nature and evolution of polyps: evaluation of the treatment and surveillance methods applied. Eur J Gastroenterol Hepatol 2009; 21: 1161-1167.

15. van Heumen BW, Nieuwenhuis MH, van Goor H, Mathus-Vliegen LE, Dekker E, Gouma DJ et al. Surgical management for advanced duodenal adenomatosis and duodenal cancer in Dutch patients with familial adenomatous polyposis: a nationwide retrospective cohort study. Surgery 2012; 151 (5): 681-690.

16. Skipworth JR, Morkane C, Raptis DA et al. Pancreaticoduodenectomy for advanced duodenal and ampullary adenomatosis in familial adenomatous polyposis. HPB (Oxford) 2011; 13: 342-349.

17. Murakami Y, Uemura K, Sasaki M et al. Duodenal cancer arising from the remaining duodenum after pylorus-preserving pancreatoduodenectomy for ampullary cancer in familial adenomatous polyposis. J Gastrointest Surg 2005; 9: 389-392.

18. Hirota WK et al. ASGE guideline: the role of endoscopy in the surveillance of premalignant conditions of the upper GI tract. Gastrointestin Endosc 2006; 63: 570-580.

19. Tianle Ma, Eun Jeong Jang, Lawrence R. Zukerberg. Recurrences are common after endoscopic ampullectomy for adenoma in the FAP syndrome. Surg Endosc 2014; 28 (8): 2349-2356.

20. Jaganmohan S, Lynch PM, Raju RP, Ross WA, Lee JE, Raju GS et al. Endoscopic management of duodenal adenomas in familial adenomatous polyposis - a single-center experience. Dig Dis Sci. 2012; 57 (3): 732-737.
21. Bettschart V, Rahman MQ, Engelken FJF, Madhavan KK, Parks RW, Garden OJ. Presentation, treatment and outcome in patients with ampullary tumors. Br J Surg 2004; 91: 1600-1607.

22. Moussata D, Senouci L, Berger F, Scoazec J-Y, Pinson S, Walter T et al. Familial Adenomatous Polyposis and Pancreatic Cancer. Pancreas: 2015; 44 (3): 512-513.

23. Pinto P, Peixoto A, Santos C, Rocha P, Pinto C, Pinheiro M et al. Analysis of Founder Mutations in Rare Tumors Associated With Hereditary Breast/Ovarian Cancer Reveals a Novel Association of BRCA2 Mutations with Ampulla of Vater Carcinomas. PLoS One 2016; 11 (8): e0161438.

24. Cordero-Fernández C, Garzón-Benavides M, Pizarro-Moreno A, García-Lozano R, Márquez-Galán JL, López Ruiz T. Gastroduodenal involvement in patients with familial adenomatous polyposis. Prospective study of the nature and evolution of polyps: evaluation of the treatment and surveillance methods applied. Eur J Gastroenterol Hepatol 2009; 21: $1161-1167$.

25. Groves CJ, Saunders BP, Spigelman AD et al. Duodenal cancer in patients with familial adenomatous polyposis (FAP): results of a 10 year prospective study. Gut 2002; 50: 636-641.

26. Latchford AR, Neale KF, Spigelman AD, Phillips RKS, Clark SK. Features of Duodenal Cancer in Patients With Familial Adenomatous Polyposis. Clin Gastroenterol Hepatol 2009;7: 659-663.

27. de Vos tot Nederveen Cappel WH, Järvinen HJ, Björk J et al. Worldwide survey among polyposis registries of surgical management of severe duodenal adenomatosis in familial adenomatous polyposis. $\mathrm{Br}$ J Surg 2003; 90: 705-710.

28. Burke C. Risk stratification for periampullary carcinoma in patients with familial adenomatous polyposis: Does theodore know what to do now. Gastroenterology 2001; 121 (5): 1246-1248.

29. Campos FG, Martinez CAR, Lopez LAB, Kanno DT, Nahas SC, Cecconello I. Advanced duodenal neoplasia and carcinoma in familial adenomatous polyposis: outcomes of surgical management. J Gastrointest Oncol 2017; 8 (5): 877-884.

30. Björk, J, Akerbrant, H, Iselius, L, Bergman, A, Engwalol, Y, Wahlstrom, J, Martinsson, T, Nordling, M, and Hultcrantz, R. Periampullary adenomas and adenocarcinomas in familial adenomatous polyposis: cumulative risks and APC gene mutations. Gastroenterology 2001; 121 : $1127-1135$. 